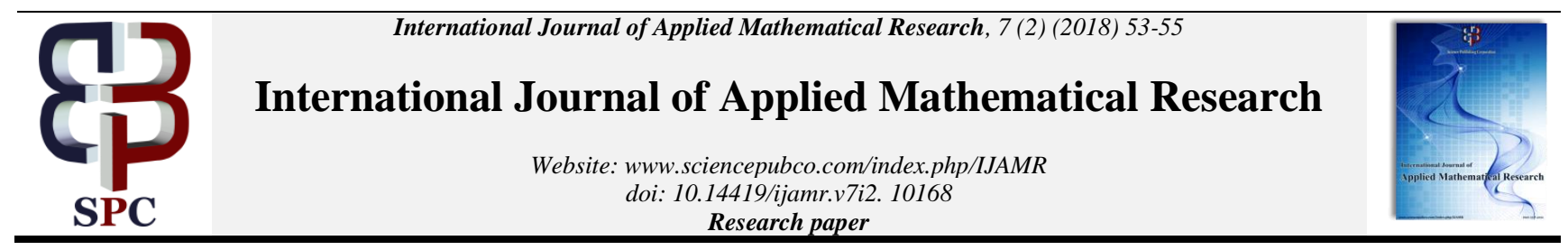

\title{
Lyapunov-Krasovskii stability analysis of nonlinear integro-differential equation
}

\author{
Jackreece P. C. * \\ Department of Mathematics /Statistics, University of port Harcourt, port Harcourt, Nigeria \\ *Corresponding author E-mail: preob.jackreece@uniport.edu.ng
}

\begin{abstract}
The purpose of this paper is to develop a qualitative stability analysis of a class of nonlinear integro-differential equation within the framework of Lyapunov-Krasovskii. We show that the existence of a Lyapunov-Krasovskii functional is a necessary and sufficient condition for the uniform asymptotic stability of the nonlinear Volterra integro-differential equations.
\end{abstract}

Keywords: Lyapunov-Krasovskii Functional, Uniform Asymptotic Stability, Integro-Differential Equation.

\section{Introduction}

Volterra integro-differential equations have wide applications in biology, ecology, medicine, physics and other scientific areas and thus has been extensively studied. Originally motivated by problems in mechanics, Mathematical biology, and Economics, the study of integro-differential and integral equations can be traced back to the works these scholars, Abel, Lotka, Fredholm, Mathlus, Verhulst and Volterra [16]. In particular, the work of Volterra on the problem of competing species was vitally important for the development of the work in this area, since then, the theory and application of Volterra integro-differential equations have emerged as an important area of research.

In the last years, a lot of interesting results related to the qualitative behaviors of solutions; stability, boundedness etc. of Volterra integro-differential equations haven obtained by many researchers, see the papers of ([1], [[2], [5], [6], [7], [9], [11], [13], [21], [22], [23], [24], [25] ) and references therein.

Several stability methods for nonlinear integro-differential systems can be found in dynamical

systems literature, such as Lyapunov-Krasovskii functionals, Razumhim, fixed point methods, Linear and nonlinear variation of parameter, stability in variation, method of reduction etc., (for detailed account see, ([4], [7], [12], [14], [15], [16]).

An important tool used in the discussion of the qualitative properties of solution of ordinary, functional and integro-differential equations is the Lyapunov's second method. The method allows one to deduce inequalities that all solutions must satisfy and from which criteria are deduced.

Over the years, Lyapunov method for the stability of integrodifferential equation have been proposed by different researcher ([8], [13], [17], [18], [19], [20], [23], [24]).

In particular [18], worked on the stability of the zero-state solution of impulsive function differential equation by applying LyapunovRazumikhin method and Piecewise continuous function to check the behavioral solution of equation. In [24], they established system's stability of a class of Volterra integro-differential equation. They used a known form of Lyapunov functional to establish the stability condition for the system. In their paper, [8] constructed a
Lyapunov functionals to check and investigate the stability for hereditary system. [23] studied certain nonlinear Volterra integro differential equations with delay. He established stability and boundedness condition of the solution by defining a suitable Lyapunov functional used to prove the result. In 2007, [19] established the stability of the solutions of a class of integro-differential equations of Volterra type whose nonlinear term is assumed to be holomorphic function of variables and possible some integral form in a small neighborhood of zero. Stability in Lyapunov's sense of single zero root and of pair of pure imaginary roots for the unperturbed equation is analyzed by relying on functional in integral form represented by Frechet series.

\section{Preliminaries}

Our aim in this paper is to use a suitable Lyapunov functional and determine necessary and sufficient condition for the stability of the zero solution of the nonlinear integro - differential equation of Volterra type defined by

$y^{\prime}(t)=B(t) g(y(t))+\int_{0}^{t} G(t, s, y(s))$

Where $y \in R$,the functions $G$ is continuous in $(t, s, y)$ for $0 \leq s \leq$ $\mathrm{t}<\infty, \mathrm{B}(\mathrm{t})$ continuous for

$0 \leq \mathrm{t}<\infty, \mathrm{g}(\mathrm{y}(\mathrm{t}))$ is continuous on $(-\infty, \infty)$ and

$\int_{0}^{t} G(t, s, y(s)) d s<\infty, \int_{0}^{t} t G(t, s, y(s)) d s<\infty$

We use the following notation and basic information throughout this paper. For any $\mathrm{t}_{0} \geq 0$ and initial function $\varphi \in\left[\mathrm{t}_{0}, \mathrm{t}\right]$, let $\mathrm{y}(\mathrm{t})=$ $y\left(t, t_{0}, \varphi\right)$ denote the solution of eq. (2.1) on $\left[t_{0}, t\right]$ such thaty $(t)=$ $\varphi(t)$. Let $C\left(\left[t_{0}, t_{i}\right]\right)$ and $C\left(\left[t_{0}, \infty\right]\right)$ denote the continuous of real valued functions on $\left[\mathrm{t}_{0}, \mathrm{t}_{1}\right]$ and $\left[\mathrm{t}_{0}, \infty\right]$ respectively. For $\varphi \in$ $\mathrm{C}\left[\mathrm{t}_{0}, 0\right],|\varphi|=\sup \left\{|\mathrm{y}(\mathrm{t})|: 0 \leq \mathrm{t} \leq \mathrm{t}_{0}\right\}$

Definition 2.1: he zero solution of eq. (2.1) is stable if for each $\varepsilon>0$ and each $t_{0} \geq 0$, there exist $\delta(\varepsilon)>0$ such that $\|\varphi\|_{t_{0}}<$ 
$\delta(\varepsilon)$ which implies that $\left\|y\left(t, t_{0}\right)\right\|<\varepsilon$, for $t \geq 0$ where $y(t, \varphi)$ is a solution of eq. (2.1) which is defined for $t \geq t_{0}$.

Definition 2.2: The zero solution of eq. (2.1) is uniformly stable if for each $\varepsilon>0$ there exist $\delta=\delta(\varepsilon)>0$ such that $\varphi \in\left[0, t_{0}\right]$ with $\|\varphi\|<\delta\left(\right.$ anyt $_{0} \geq 0$ ) implies that $\|y(t, \varphi)\|<\varepsilon$ for all $t \geq$ $t_{0}$

Definition 2.3: The zero solution of eq. (2.1) is said to be asymptotically stable if it is stable and there is a number $\delta>0$ such that any solution $y(t)$ with $\|\varphi\|<\delta$ satisfies $\lim _{t \rightarrow \infty}\|y(t)\|=0$.

The following theorem is essential for stability result and is a basic tool for our results.

Theorem 2.1: [9]. If there exists a functionalV $(t, \varphi()$.$) , defined$ whenever $t \geq t_{0} \geq 0$ and $\varphi \in C\left([0, t], R^{n}\right)$ such that

i) $\quad \mathrm{V}(\mathrm{t}, 0) \equiv \mathrm{o}$, Vis continuous in $\mathrm{t}$ and locally Lipschitz in $\varphi$

ii) $\mathrm{V}(\mathrm{t}, \varphi(\mathrm{t})) \geq \mathrm{W}(|\varphi(\mathrm{t})|), \mathrm{W}:[0, \infty) \rightarrow[0, \infty)$ is a continuous function with $\mathrm{W}(0)=0, \mathrm{~W}(\mathrm{r})>0$, if $\mathrm{r}>0$ and $\mathrm{W}$ is strictly increasing (positive definiteness), and

iii) $\mathrm{V}^{\prime}(\mathrm{t}, \varphi()) \leq$.

Then the zero solution of eq. (2.1) is stable and

$\mathrm{V}(\mathrm{t}, \varphi())=.\mathrm{V}(\mathrm{t}, \varphi(\mathrm{s})): 0 \leq \mathrm{s} \leq \mathrm{t}$

Is called a Lyapunov function of eq. (2.1)

Theorem 2.2: (Lyapunov-Krasovskii stability) Suppose that $f: R \times C \rightarrow R^{n}$ given in eq. 2.1 map every $\{R \times(\ldots)\} R \times$ (bounded in $C$ )into a bounded set in $R^{n}$, and that $u, v, w: R_{+} \rightarrow$ $R_{+}$are continuously non decreasing functions, where additionally $u(s)$ and $v(s)$ are positive for $s>0$ and $u(0)=0$. If there exists a continuous differentiable function $V: R \times C \rightarrow R$ such that $u(\|\phi(0)\|) \leq V(t, \phi) \leq v\left(\|\phi\|_{C}\right)$ and $V^{\prime}(t, \phi) \leq-w(\|\phi(0)\|)$ then the trivial solution of eq. 2.1 is uniformly stable. Ifw $(s)>$ 0 for $s>0$, then it is uniformly asymptotically stable. If in addition $\lim u(s)=\infty$, then it is globally uniformly asymptotically stable, [12].

\section{Main result}

Theorem 3.1: If $B(t)<0, G(t, s, y(s))>0$ and

$$
B(t) g(y(t))+\int_{0}^{t} G(t, s, y(s)) d s \neq 0
$$

Then the statements below are equivalent.

i) The solution of eq. (2.1) tends to zero.

ii) $\mathrm{B}(\mathrm{t}) \mathrm{g}(\mathrm{y}(\mathrm{t}))+\int_{0}^{\mathrm{t}} \mathrm{G}(\mathrm{t}, \mathrm{s}, \mathrm{y}(\mathrm{s})) \mathrm{ds}<-\xi, \xi>0$

iii) Every solution of (2.1) is a Lebesgue integrable function with respect to the vector space $\mathrm{R}^{\mathrm{n}}$. For the proof see, [13].

Theorem 3.2: Suppose $m, n: R^{n} \rightarrow R^{+}$are continuous increasing function, $m(s)$ and $n(s)$ are positive for $s \geq 0, m(0)=$ $n(0)=0$. suppose there exist a function $V(t, s, y(s))$ satisfying the following condition.

$\mathrm{C} 1: \mathrm{m}(|\mathrm{s}|) \leq \mathrm{V}(\mathrm{t}, \mathrm{s}, \mathrm{y}(\mathrm{s})) \leq \mathrm{n}(|\mathrm{s}|)$

C2: $V^{\prime}\left(t, s y,\left(t, s_{0}, t_{0}\right)\right) \leq 0$ for $t>t_{0}$.

Then the steady sate solution $y=0$ is uniformly stable Proof

Given $\varepsilon>0$ such that $|y| \leq \varepsilon$.since $V(t, s, y(s))$ is positive definite and continuous, then the function $\mathrm{m}$ and $\mathrm{n}$ satisfy conditionC1, that is $\mathrm{m}(|\mathrm{s}|) \leq \mathrm{V}(\mathrm{t}, \mathrm{s}, \mathrm{y}(\mathrm{s})) \leq \mathrm{n}(|\mathrm{s}|)$ for $\mathrm{t} \in$ $\mathrm{R}$ and $\mathrm{y} \in \mathrm{R}^{+}$.Taking $\delta(\varepsilon)>0$ such that $0<\delta(\varepsilon)<$ eand $\mathrm{m}(\varepsilon)<\mathrm{n}(\delta)$

Assume that $\left|\mathrm{y}_{0}\right| \leq \delta$

$\mathrm{y}\left(\mathrm{t}, \mathrm{s}_{0}, \mathrm{y}_{0}\right)=\mathrm{y}(\mathrm{t})$ Being the solution of eq. (2.1) and

$\left|\mathrm{y}\left(\mathrm{t}, \mathrm{s}_{0}, \mathrm{y}_{0}\right)\right|<\varepsilon$, for $0 \leq \mathrm{t}<\mathrm{t}_{2}$, so thatm $(\varepsilon) \leq$

$\mathrm{V}\left(\mathrm{t}_{2}, \mathrm{~s}_{2}, \mathrm{y}\left(\mathrm{t}_{0}\right)\right)$. From condition $\mathrm{C} 1$, it means that $\mathrm{y}\left(\mathrm{t}, \mathrm{s}_{0}, \mathrm{y}_{0}\right)$ is a solution for $0 \leq t<t_{2}$.If the estimate,

$\mathrm{V}\left(t_{s}, y\left(t_{s}\right) \leq V\left(0, y_{0}\right)\right)$

Now using $\mathrm{C} 1$ and our choice of $\delta(\varepsilon)$, leads to the contradiction,

$m(\varepsilon) \leq V\left(t_{2}, s_{2}, y\left(t_{0}\right) \leq V\left(0, s_{0}, y_{0}\right) \leq n\left(\left|s_{0}\right|\right) \leq m(\varepsilon)<\right.$ $n(\delta)$.

Since mis increasing, we have that $\left|y\left(t_{2}\right)\right|<\varepsilon$, a contradiction. Then,

$V\left(t_{2}, s_{2}, y\left(t_{0}\right) \geq V\left(0, s_{0}, y_{0}\right)\right.$

Since $V(t, s, y)$ is continuous in $\mathrm{t}$, there exist $\mathrm{t}$ such that $0<t_{1}<$ $t_{2}$. this implies that

$V(t, s, y(t)) \leq V\left(t_{1}, s_{1}, y\left(t_{1}\right)=V\left(t_{2}, s_{2}, y\left(t_{2}\right)\right.\right.$ for $0 \leq t<$ $t_{1}$.

If there exist a sequence $\alpha_{1}$ for $o \leq \alpha_{i}<t_{1}$ such that $i \rightarrow$ $\infty$,then

$V^{\prime}\left(\alpha_{i}, s_{i} y\left(t_{i}\right)\right)>0$, for $i=0,1,2$,

Assuming the derivative above is not true, then $V^{\prime}(t, s, y(t)) \leq 0$ for all $t_{i}$.

Hence,

$V\left(t_{1}, s_{1}, y\left(t_{1}\right) \leq V(t, s, y(t))\right.$

This completes the proof of uniform stability.

Theorem 3.3: Assuming all the conditions of theorem (3.2) are satisfied and in addition $q(s)>0$ if $s>0$. If condition $C 2$, is strengthened to $V^{\prime}(t, s, y(t)) \leq-q(|y(t)|)$ for $t>0$. Then the solution $y=0$ of eq. (2.1) is uniformly asymptotically stable.

Proof Continuing with the proof of theorem (3.2), we need to show that the solution $y=0$ of eq. (2.1) is uniformly asymptotically stable. Let $y(t)=y(t, s, y)$ be any solution of equation (2.1) which is bounded on $[0, \infty)$. We may define

$\operatorname{Sup}|y(t)|=\delta_{i}$

$t \geq 0$

Then,

$m(\varepsilon)<n\left(\delta_{i}\right)$

Assuming that $P(V(t, s, y(t)))>V(s, y(s))$ for $0 \leq s \leq t$ Which is continuous for $\mathrm{s}>0$, if there exist a number $\alpha=$ $\alpha(\varepsilon)>0$ such that $s+\alpha<p(s)$ For $n\left(\delta_{i}\right) \leq s \leq m(\varepsilon)$.Also if there exist a positive integer $N(\varepsilon)=N>0$ such that $n\left(\delta_{i}\right)-N \alpha \leq m(\varepsilon)$ for some $t \geq 0$, we have $V(t, s, y(t)) \geq$ $m(\varepsilon)$. It follows that $\delta_{j}=\delta_{j}(\varepsilon)>0$ such that $|y(t)|>\delta_{j}$.

This implies from $\mathrm{C} 1$ of theorem 3.2 that $q(y(t)) \geq q\left(\delta_{j}\right) \equiv$ $\delta_{k}$ It is evident that $\overline{\delta_{k}}$ rely on $\varepsilon$. We can define a number $t_{p}=$ $t_{s}\left(t_{0}, \varepsilon\right)$ such that $t_{0}\left(t_{0}, \varepsilon\right)=t_{0}, t_{p+1}\left(t_{0}, \varepsilon\right)+\frac{\alpha}{\delta_{k}}$, this then gives $t_{p}\left(t_{0}, \varepsilon\right)=t_{0}+s \frac{\alpha}{\delta_{k}}$. Assuming $u(\varepsilon)=$ 
$N\left(\frac{\alpha}{\delta_{k}}\right)$, then $t_{N}\left(t_{0}, \varepsilon\right)=t_{0}+u(\varepsilon)$. To prove the uniform asymptotic stability, we must prove that,

$\left|y\left(t, s_{0}, y_{0}\right)\right|<\varepsilon f$ or $t \geq t_{o}+u(\varepsilon)$ where $\left|y_{0}\right|<\delta_{i}$

Suppose, $\quad V(t, s, y(t))<m(\varepsilon)+(N-P) \alpha, t \geq t_{s}$ for $p=$ $0,1,2, \ldots, N$. For $p=0$, we have $V(t, s, y(t))<m(\varepsilon)+N \alpha$, and for $p=1, V(t, s, y(t))<m(\varepsilon)+(N-1) \alpha$, hence for some $p$, we have $V(t, s, y(t))<m(\varepsilon)+(N-P) \alpha, s \geq t_{p}$. This possibly gives the inequality

$$
V(t, s, y(t)) \geq m(\varepsilon)+(N-P-1) \alpha, t \in\left[t_{p}, t_{p+1}\right]
$$

Which follows from $\mathrm{C} 1$ of theorem 3.2 that $m(\varepsilon) \leq$

$V(t, s, y(t)) \leq n\left(\delta_{i}\right)$, here, we can show that

$V(t, s, y(t))<m(\varepsilon)+(N P-1) \alpha \leq V(t, s, y(t))+\alpha$

$<P(V(t, s, y(t)))$ for $t_{p} \leq s \leq t, t \in\left[t_{p}, t_{p+1}\right]$

We can also obtain

$$
\begin{aligned}
& V\left(t_{p+1}, s_{p+1}, y\left(t_{p+1}\right)\right) \leq V\left(t_{p}, s_{p}, y\left(t_{p}\right)\right) \\
& -\int_{p}^{p+1} q(|y(s)|) d s<m(\varepsilon)+(N-p) \alpha-\delta_{k}\left(t_{p+1}-t_{p}\right) \\
& <m(\varepsilon)+(N-p) \alpha<V\left(t_{p}, s_{p}, y\left(t_{p}\right)\right)
\end{aligned}
$$

This contradiction shows that if there exist $t^{\prime} \in\left[t_{p}, t_{p+1}\right]$, then

$V(t, s, y(t)) \geq m(\varepsilon)+(N-P-1) \alpha$

This implies that

$V(t, s, y(t)) \geq m(\varepsilon)+(N-P-1) \alpha$

Assuming it is false, given $t_{1}>t^{\prime}$ such that $V\left(t^{1}, s_{1}, y\left(t_{1}\right)\right)<$ $m(\varepsilon)+(N-P-1) \alpha$, for an integerw $<$ $0, V\left(t_{1},+w, s_{1}, y\left(t_{1}+w\right)\right)<m(\varepsilon)+(N-P-1) \alpha$. Then we have

$V^{\prime}\left(t_{1}, s_{1} y\left(t_{1}\right)\right) \geq 0$

Assuming it is not true, we prove that $V(t, s, y(t)) \leq$ $P(V(t, s, y(t)))$ for $\mathrm{t}^{\prime} \leq \mathrm{s} \leq \mathrm{t}_{1}$ and so we have $\mathrm{V}^{\prime}\left(\mathrm{t}_{1}, \mathrm{~s}_{1}, \mathrm{y}\left(\mathrm{t}_{1}\right)\right) \leq-\mathrm{q}(|\mathrm{y}(\mathrm{t})|) \leq-\delta_{\mathrm{k}}<0$, which shows the asymptotic stability and $\mathrm{V}(\mathrm{t}, \mathrm{s}, \mathrm{y}(\mathrm{t}))<\mathrm{m}(\varepsilon)+(\mathrm{N}-\mathrm{P}-$ 1) $\alpha$ for $t>t_{p+1}$, and also contradicts

$\mathrm{V}^{\prime}(\mathrm{t}, \mathrm{s}, \mathrm{y}(\mathrm{t})) \geq 0$

This completes the proof.

\section{Conclusion}

A kind of nonlinear Volterra integro-differential equation has been considered. By defining an appropriate Lyapunov-Krasovskii functional some asymptotic stability/uniform asymptotic stability results has been discussed which extends known results in the literature.

\section{References}

[1] Adivar M. and Raffoul Y. N., Inequalities and exponential stability in finite delay Volterra integro- differential equations, Rend. Circ

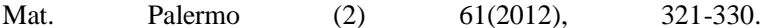
https://doi.org/10.1007/s12215-012-0092-4.

[2] Becker L. C., Uniform continuous L1 - solution of Volterra equations and global asymptotic stability, Cubo 11(2009), 1-24.

[3] Burton T. A., Volterra integral and differential equations Second Edition, Mathematics in Science and Engineering, 202, Elsevier B. V. Amsterdam, 2005

[4] Burton T. A., Stability theory for Volterra equations, J. Differential Equations 32(1979), 1, 101-118.

[5] Burton T. A. and Mahfoud W. E., Stability criteria for Volterra Equations, Trans. Amer. Soc. 279 (1983), 1, 143-174.

[6] Burton T. A. and Haddock J. R., Qualitative properties of solutions of integral equations, Nonlinear Anal. 71 (2009), No. 11, 5712 5723.

[7] Burton, T., Volterra integral and Differential Equations, Elsevier B V., Amsterdam, 2005.

[8] Caraballo, T., Real, J. and Shaikhet, L., Method of Lyapunov functional construction in stability of delay evolution equation. J. Math Anal. $334 . \quad$ (2007):1130-1145. https://doi.org/10.1016/j.jmaa.2007.01.038.

[9] Davies I., Nwoaburu A. O., Jackreece P. C.; Criteria for Asymptotic Stability for Linear Delay Systems of the Volterra Type, Journal of The Mathematical Association of Nigeria, (Abacus), Vol. 32 \#2A, pp 101-109, 2005.

[10] Driver, R., Existence and stability of solution of a delayDifferential system, Arch. Rat. Mech. Anal., 10, (1962):401-426. https://doi.org/10.1007/BF00281203.

[11] Eloe P., Islam M. and Zhang B., Uniform asymptotic stability of Linear Volterra integro-differential equations with application to Delay systems, Dynam. Systems Appl. 9(200), No. 3, 331-344.

[12] Hale, J. K. and Lunel, S. M. V., Introduction to Functional Differential Equations, Springer-Verlag, New York, 1993. https://doi.org/10.1007/978-1-4612-4342-7.

[13] Jackreece, P. C., and Aniaku, S., Stability Results of Nonlinear Integro-differential Equations. Mathematical Theory and Modeling, 8(1), (2018), 27-33.

[14] Kuang, Y., Delay Differential Equations with Applications in Population Dynamics, Academic Press Inc., San Diego, 1993.

[15] La Salle, J. and Lefschetz S., Stability by Lyapunov's direct method with applications, New York Academic Press, 1961.

[16] Lakshmikanthan, V. and Rao, M. R. M., Theory of Integrodifferential Equations, Gordon and Breach Science Publishers, Amsterdam, 1995.

[17] Stamova, I. M., and Stamov, G. T., Analysis of differential equation with maximum, Math. Slovaca, 63(6), (2013): 1291-1302. https://doi.org/10.2478/s12175-013-0171-9.

[18] Stamov, I. M., and Stamov, G., T., Lyapunov-Razumikhim method for impulsive functional differential equations and application to population dynamics, J. compt. Appl. Math. 130, (2001):163-171

[19] Sergeev, V. S., Stability of solutions of Voterra integro-differential equations Mathematical and computer modeling. 45, (2007):13761394. https://doi.org/10.1016/j.mcm.2006.09.023

[20] Tunc, C. A note on the qualitative behaviors of nonlinear Volterra integro-differential equation, Journal of the Egyptian Mathematical Society, 24, (2016), 187-192. https://doi.org/10.1016/j.joems.2014.12.010.

[21] Tunç, C., and Abid, S. (2017). A remark on the stability and boundedness criteria in retarded Volterra integro-differential equations, Journal of the Egyptian Mathematical Society, 25, 363-368. https://doi.org/10.1016/j.joems.2017.05.001.

[22] Tunc, C., New Stability and boundedness results to Volterra integro-differential equations with delay, Journal of the Egyptian $\begin{array}{lll}\text { Mathematical } & \text { Society, 21016): }\end{array}$ https://doi.org/10.1016/j.joems.2015.08.001.

[23] Tunç, C. (2016). Qualitative properties in nonlinear Volterra integro-differential equations with delay. Journal of Taibah University for Science. ps://doi.org/10.1016/j.jtusci.2015.12.009.

[24] Vanualailai, J. and Nakagiri, S., Stability of a System of Volterra integro-differential equations. J. Math. Anal. Appl., Vol. 281(2), 2003, 602-619. https://doi.org/10.1016/S0022-247X(03)00171-9.

[25] Wazwaz, A. M., Linear and nonlinear integral equations, methods and applications, Higher Education Press, Beijing, Springer, Heidelberg, 2011. https://doi.org/10.1007/978-3-642-21449-3. 